\title{
Application of Indocyanine Green (ICG)
}

Fluorescence imaging technique using indocyanine green (ICG) has been developed, and well used to define anatomic structures and define segments to be resected.

\section{- Fluorescence liver mapping}

ICG is injected intravenously, after which it binds to plasma proteins and remains in the vascular space until selective uptake by the liver and excretion into the bile. Its unique catabolic character makes ICG particularly suitable for visualizing anatomical structures in the liver. Therefore, ICG can be used for anatomic landmark for resection.

- Fluorescence cholangiography

ICG also can be used to identify structures of biliary system. It may help for guiding transection of bile duct in donor surgery. Fluorescent images of the biliary tract can be obtained by intrabiliary and intravenous injection of ICG. For intrabiliary injection, the fluorescence intensity of protein-bound ICG was found to correlate with its concentration. Thus, to obtain clear fluorescence images of the bile ducts diluted ICG solution should be used for imaging. It is also important to aspirate a small amount of bile into the syringe before injection to promote binding of ICG to proteins. Intrahepatic bile duct anatomy and the extrahepatic biliary system can be identified.

- Fluorescence imaging of hepatic tumors

The fluorescence pattern in hepatic tumors could be classified into three types: total fluorescence, in which all tumor tissue showed uniform fluorescence; partial fluo- rescence, in which some tumor tissues showed fluorescence; and rim fluorescence, in which the cancer tissues were negative for fluorescence, but the surrounding liver parenchyma showed fluorescence. These fluorescence patterns were closely associated with the characteristics of the liver cancers. Total fluorescence-type tumors included all well-differentiated hepatocellular carcinoma (HCC)s, whereas rim fluorescence type tumors consisted only of poorly differentiated HCCs and colorectal liver metastasis (CRLM).

The intraoperative ICG-fluorescence imaging of hepatic tumors is simple and is especially useful for identifying subcapsular lesions for removal during laparoscopic hepatectomy, in which visual inspection and palpation are limited compared with open surgery.

\section{Suggested Readings}

Mitsuhashi N, Kimura F, Shimizu H, Imamaki M, Yoshidome H, Ohtsuka M, et al. Usefulness of intraoperative fluorescence imaging to evaluate local anatomy in hepatobiliary surgery. J HepatoBiliary-Pancreat Surg. 2008;15:508-14.

Rosenthal EL, Warram JM, de Boer E, Basilion JP, Biel MA, Bogyo M, et al. Successful translation of fluorescence navigation during oncologic surgery: a consensus report. J Nucl Med. 2016;57(1):144-50.

Terasawa M, Ishizawa T, Mise Y, Inoue Y, Ito H, Takahashi Y, et al. Applications of fusion-fluorescence imaging using indocyanine green in laparoscopic hepatectomy. Surg Endosc. 2017;31:5111-8. 